\title{
Objective assessment of cosmetic outcome after targeted intraoperative radiotherapy in breast cancer: results from a randomised controlled trial
}

\author{
Mohammed R. S. Keshtgar - Norman R. Williams • Max Bulsara • \\ Christobel Saunders $\cdot$ Henrik Flyger $\cdot$ Jaime S. Cardoso $\cdot$ Tammy Corica \\ Neils Bentzon • Nikolaos V. Michalopoulos $・$ David J. Joseph
}

Received: 19 March 2013/ Accepted: 10 July 2013/Published online: 23 July 2013

(C) Springer Science+Business Media New York 2013

\begin{abstract}
The international randomised targeted intraoperative radiotherapy (TARGIT) trial has demonstrated evidence of non-inferiority between the novel technique of TARGIT (intra-operative radiotherapy with Intrabeam $^{\circledR}$ ) and conventional external beam radiotherapy (EBRT) in women with early breast cancer in terms of the primary outcome measure of risk of local relapse within the treated breast. Cosmesis is an increasingly important outcome of breast conserving treatment with both surgery and radiotherapy contributing to this. It was unknown if the single high dose of TARGIT may lead to damaging fibrosis and thus impair cosmesis further, so we objectively evaluated the aesthetic outcome of patients within the TARGIT randomised controlled trial. We have used an objective assessment tool for evaluation of cosmetic outcome.
\end{abstract}

Electronic supplementary material The online version of this article (doi:10.1007/s10549-013-2641-8) contains supplementary material, which is available to authorized users.

M. R. S. Keshtgar $(\bowtie) \cdot$ N. V. Michalopoulos

Department of Surgery, Royal Free London NHS Foundation

Trust, University College London, Pond Street, London NW3

2QG, UK

e-mail: m.keshtgar@ucl.ac.uk

N. R. Williams

Clinical Trials Group, Centre for Clinical Science and Technology, UCL Medical School, Clerkenwell Building, Archway Campus, Highgate Hill, London N19 5LW, UK

\section{Bulsara}

Institute of Health and Rehabilitation Research, University of Notre Dame, 19 Mouat Street, P.O. Box 1225, Fremantle, WA 6959, Australia

C. Saunders

School of Surgery, QEII Medical Centre, Perth, WA 6009, Australia
Frontal digital photographs were taken at baseline (before TARGIT or EBRT) and yearly thereafter for up to 5 years. The photographs were analysed by BCCT.core, a validated software which produces a composite score based on symmetry, colour and scar. 342 patients were assessed, median age at baseline 64 years (IQR 59-68). The scores were dichotomised into Excellent and Good (EG), and Fair and Poor (FP). There were statistically significant increases in the odds of having an outcome of EG for patients in the TARGIT group relative to the EBRT group at year 1 (OR $2.07,95 \%$ CI $1.12-3.85, p=0.021)$ and year 2 (OR 2.11, $95 \%$ CI $1.0-4.45, p=0.05)$. Following a totally objective assessment in a randomised setting, the aesthetic outcome of patients demonstrates that those treated with TARGIT have a superior cosmetic result to those patients who received conventional external beam radiotherapy.

Keywords Breast cancer - Intra-operative radiotherapy · TARGIT $\cdot$ Cosmesis

H. Flyger · N. Bentzon

Department of Surgery, Herlev Hospital, University

of Copenhagen, 2730 Herlev, Denmark

J. S. Cardoso

INESC TEC and Faculty of Engineering, University of Porto,

Porto, Portugal

T. Corica

Radiation Oncology, Sir Charles Gairdner Hospital, Nerdland,

WA, Australia

D. J. Joseph

Sir Charles Gairdner Hospital, Hospital Avenue, Nedlands, WA 6009, Australia 


\section{Introduction}

Breast cancer is a global problem and its incidence has had an annual rate of increase of $3.1 \%$ from 1980 to 2010 [1]. During this time, the surgical treatment of early breast cancer has moved from radical to minimally invasive; that is, from mastectomy to breast conserving surgery. The benefits of this approach have been proven in randomised controlled trials [2, 3].

However, radiotherapy treatment continues to involve irradiation of both the tumour bed and the whole breast. Recent data from the randomised controlled trial of targeted intraoperative radiotherapy (TARGIT) has confirmed that, in selected women with early breast cancer, the technique of intra-operative radiotherapy (IORT) using the TARGIT technique is safe and as effective as conventional external beam radiotherapy to the whole breast [4]. In this approach, therapeutic irradiation is delivered to the tumour bed from within the breast after wide local excision using a portable radiotherapy device.

With improvements in survival of patients with breast cancer [5], the aesthetic outcome has become an increasingly important consideration. Radiotherapy can cause significant differences in colour such as hyperpigmentation of the breast, hypopigmentation of the nipple-areola complex and telangiactesia [6]. Moreover, surgery and radiotherapy-associated fibrosis can impact on symmetry without impairing the size of the breast by causing upward retraction of the inferior mammary sulcus and/or the nipple-areola complex [7]. A variety of methods for determining cosmetic outcome have been used. A subjective, overall cosmetic score classifying outcome as excellent, good, fair and poor was described in 1979 by Harris et al. [8]. The first attempt at an objective measurement of the cosmetic result after breast surgery was the breast retraction assessment (BRA) method introduced in 1985 by Pezner et al. [7]. In 2007, two groups took this approach further and developed software using digital images of patients to evaluate the cosmetic results of breast conservative treatment. Fitzal et al. [9] applied a Breast Analysing Tool (BAT) which analysed symmetry differences from both breasts. Cardoso et al. [10] developed a software programme Breast Cancer Conservative Treatment. cosmetic results (BCCT.core) which combines various objective measures of asymmetry, skin colour and scar, resulting in an overall cosmetic score based on the classification system of Harris et al. [8] which was subsequently validated [11]. BCCT.core allows reproducible, objective aesthetic evaluation of breast cancer conservative treatment from digital photographs [11].

We used BCCT.core to objectively evaluate the aesthetic outcome of patients within the TARGIT randomised controlled trial.

\section{Materials and methods}

Patients enrolled from two centres participating in the TARGIT randomised trial who underwent breast conserving surgery and radiotherapy were included in this study. Patients were randomized to receive either IORT by the Intrabeam $^{\circledR}$ (Carl Zeiss, Germany) device (20 Gy to surface of tumour bed equivalent to 5-6 Gy at depth of $1 \mathrm{~cm}$ from surface of the applicator) or the conventional 3-5 week external beam radiotherapy as per local protocol. Patients' demographics and tumour characteristics are summarised in Table 1.

The TARGIT technique has been described earlier [4]. Briefly, the entire radiation therapy is delivered to the tumour bed in a single fraction at the time of surgery, following the removal of the breast cancer. An applicator of appropriate size is placed directly into the lumpectomy cavity and radiotherapy is given to the tissue which is most susceptible to the tumour recurrence, while sparing the surrounding tissue away from the tumour from the effects of radiation. TARGIT can be given either pre-pathology (at the same time as the wide local excision) or post-pathology (where the wide local excision is performed first, then TARGIT given in a subsequent procedure after histopathology review). All patients in this study were in the postpathology cohort.

When the TARGIT trial patients were willing to provide consent to have photographs taken for the cosmesis study, digital photographs were taken according to the written protocol at baseline (prior to TARGIT or EBRT) and yearly thereafter for up to 5 years. Frontal views were used to analyse the cosmetic outcome by applying the BCCT.core software (BCCT.core 2.0, INESC Porto, Portugal) (Fig. 1).

BCCT.core introduces user-independent evaluation of results preceded by automatic localisation of fiducial points (nipples, breast contour and sternal notch) on digital photographs. It objectively evaluates asymmetry, colour change and scar. Asymmetry between breasts is evaluated using several indices (e.g. breast retraction assessment, difference in lower breast contour levels, upward nipple retraction, breast compliance evaluation, breast contour length difference, breast area difference and breast overlap difference). To extract colour features of each breast, a histogram analysis is carried out followed by an evaluation of dissimilarity. Scar visibility is translated into local colour dissimilarity, by comparing corresponding breast sectors [11]. All measures of individual aesthetic characteristics are automatically assessed and reported. Results are translated according to Harris scale categories: Excellent (treated breast nearly identical to untreated breast), Good (treated breast slightly different from untreated breast), Fair (treated breast clearly different from 
Table 1 Patient, tumour and treatment characteristics

\begin{tabular}{|c|c|c|}
\hline Parameter & TARGIT & EBRT \\
\hline \multicolumn{3}{|l|}{ Patient characteristics } \\
\hline Number of patients in this study & 178 & 164 \\
\hline From Perth, Australia & 51 & 53 \\
\hline From Copenhagen, Denmark & 127 & 111 \\
\hline $\begin{array}{l}\text { Age at randomisation (years), } \\
\text { mean (SD) }\end{array}$ & $63.6(6.7)$ & $63.2(6.3)$ \\
\hline Height $(\mathrm{cm})$ mean, (SD) & $166(6.1)$ & $165(5.7)$ \\
\hline Weight (kg) mean, (SD) & $72.3(12.8)$ & $72.3(14.3)$ \\
\hline $\begin{array}{l}\text { Body mass index }\left(\mathrm{kg} / \mathrm{m}^{2}\right) \\
\text { mean }(\mathrm{SD})\end{array}$ & $26.4(4.7)$ & $26.7(5.3)$ \\
\hline \multicolumn{3}{|l|}{ Tumour characteristics } \\
\hline Specimen weight $(\mathrm{g})$ mean, $(\mathrm{SD})$ & $40.5(25.1)$ & $37.4(19.7)$ \\
\hline Tumour size (mm) mean, (SD) & $10.3(4.8)$ & $10.6(4.1)$ \\
\hline \multicolumn{3}{|l|}{ Laterality } \\
\hline Right $(\%)$ & $89(50)$ & $80(49)$ \\
\hline Left $(\%)$ & $89(50)$ & $84(51)$ \\
\hline \multicolumn{3}{|l|}{ Screen detected } \\
\hline Yes $(\%)$ & $123(69)$ & $110(67)$ \\
\hline No $(\%)$ & $55(31)$ & $54(33)$ \\
\hline \multicolumn{3}{|l|}{ Tumour grade } \\
\hline $1(\%)$ & $118(66)$ & $106(65)$ \\
\hline $2(\%)$ & $40(23)$ & $38(23)$ \\
\hline $3(\%)$ & $3(2)$ & $6(4)$ \\
\hline Unknown (\%) & $17(10)$ & $14(9)$ \\
\hline \multicolumn{3}{|l|}{ Treatment } \\
\hline \multicolumn{3}{|l|}{ Incision type } \\
\hline Circumferential (\%) & $113(65)$ & $93(58)$ \\
\hline Transverse $(\%)$ & $27(16)$ & $17(11)$ \\
\hline Circumareolar (\%) & $7(4)$ & $11(7)$ \\
\hline Radial (\%) & 27 (16) & $38(24)$ \\
\hline Chemotherapy: no (\%) & $178(100)$ & $164(100)$ \\
\hline \multicolumn{3}{|l|}{ Adjuvant hormonal therapy } \\
\hline Yes $(\%)$ & $72(40)$ & $74(45)$ \\
\hline No $(\%)$ & $106(60)$ & $90(55)$ \\
\hline \multicolumn{3}{|l|}{ Follow-up } \\
\hline $\begin{array}{l}\text { Median follow-up (years) } \\
\text { (interquartile range) }\end{array}$ & $2.0(1.1-2.7)$ & $2.0(1.1-2.7)$ \\
\hline
\end{tabular}

untreated breast, but not seriously distorted) and Poor (treated breast seriously distorted) [8] (Fig. 2).

Our study has been planned and executed in line with the declaration of Helsinki (2002), and local ethical approvals were in place before commencement. Written informed consent was obtained by all patients prior to enrolment in the study.

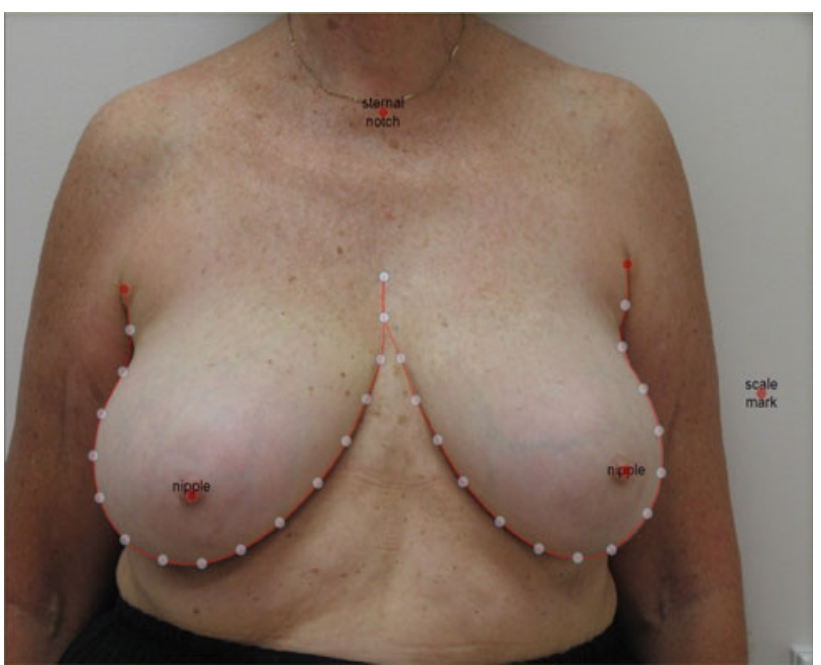

Fig. 1 BCCT.core software in use

Some photographs could not be assessed for technical reasons such as poor lighting or the presence of dressings, those who withdrew consent for further follow-up and patients for whom there was not at least one assessable photograph at year one or later. Other exclusions included: previous contralateral breast cancer $(n=25)$, presence of blue dye from sentinel lymph node $(n=3)$, mastectomy $(n=2)$, breast reconstruction $(n=2)$, recurrent disease $(n=2)$ and one patient who died.

Statistical analysis

Analysis was according to treatment received (TARGIT or EBRT).The cosmetic outcome scores were dichotomised into Excellent and Good (EG), and Fair and Poor (FP). A generalised estimating equation (GEE) regression model was used to compare the cosmetic outcome scores between treatment groups. An additional analysis was done controlling for confounding variables (tumour size, tumour grade and age of the patient), as these factors have been recognised to influence cosmetic results after conservation therapy for breast cancer [12]. For each year of assessment, a logistic regression model was used to compare the cosmetic outcome scores between treatment groups controlling for confounding variables. Odds ratios and $95 \%$ confidence intervals for the odds ratios are presented. For the component BCCT indices, a delta measure (difference between baseline and each subsequent visit) was calculated for each treatment group. The comparison of treatment group for each delta was conducted using Student's $t$ test. The trend for change over time for each delta was assessed using linear mixed model, defining treatment, time and interaction as fixed effects and the patient as a random effect. 
Fig. 2 Examples of cosmetic assessment *Excellent (treated breast nearly identical to untreated breast), Good (treated breast slightly different from untreated breast), Fair (treated breast clearly different from untreated breast, but not seriously distorted) and Poor (treated breast seriously distorted)

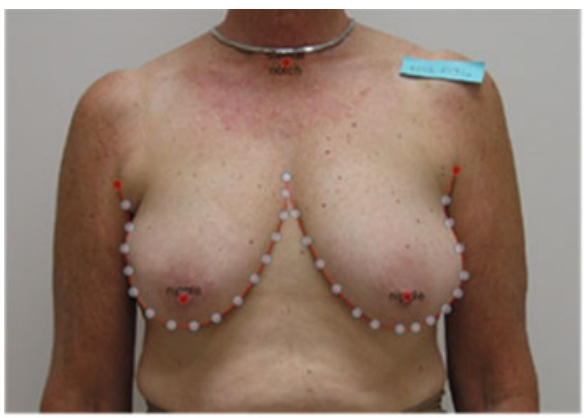

Excellent

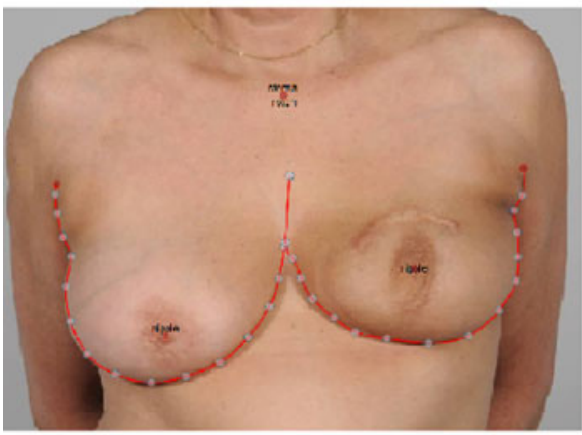

Fair

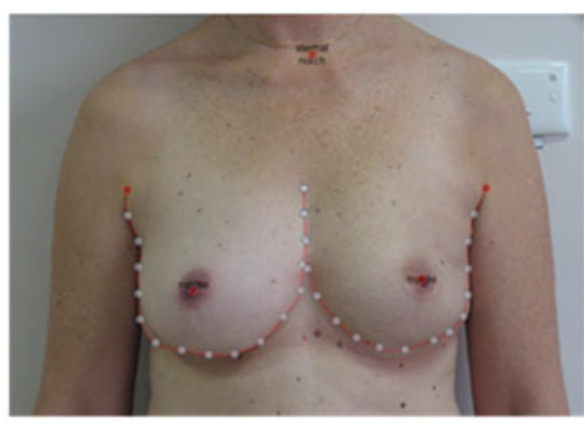

Good

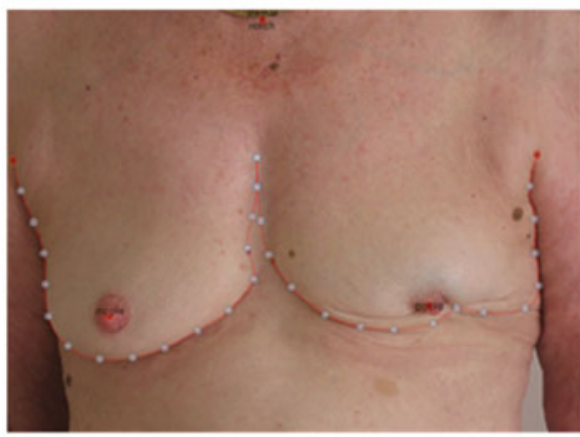

Poor

\section{Results}

Photographs were available from 342 patients. Baseline patient and tumour characteristics, and the location of tumours, were similar between the groups (Table 1; Fig. 3). There was an overall $61 \%$ increase in the odds of having an outcome of EG for patients in the TARGIT group relative to the EBRT group (OR 1.61, $95 \% \mathrm{CI}$ $1.0-2.60, p=0.05$ ) which increased to $63 \%$ (OR 1.63, $95 \%$ CI 1.01-2.64, $p=0.046$ ) after adjusting for age of the patient, tumour size and grade. At year one, there was a statistically significant 2.07-fold increase in the odds of having an outcome of EG for patients in the TARGIT group relative to the EBRT group (OR 2.07, $95 \% \mathrm{CI}$ $1.12-3.85, p=0.021$ ) which became 2.01 (OR 2.01, $95 \%$ CI $1.08-3.74, p=0.029$ ) after adjusting for age of the patient, tumour size and grade. Similarly, for year 2, there was a statistically significant 2.11-fold increase in the odds of having an outcome of EG for patients in the TARGIT group relative to the EBRT group (OR 2.11, $95 \% \mathrm{CI}$ $1.0-4.45, p=0.05$ ) which increased to 2.48 (OR 2.48, $95 \%$ CI 1.16-5.30, $p=0.020$ ) after adjusting for age of the patient, tumour size and grade. The overall scores over time in each of the two treatment groups are illustrated in Fig. 4a.

A detailed analysis of the component indices used to compile the overall score indicated significant differences in some measures (see Web Table 1). One measure of asymmetry, lower breast contour (Fig. 4b), was greater in the EBRT arm throughout the whole follow-up period of five years. Significant differences in a measure of colour (cX2b, see Fig. 4c) and scar (sEMDb, Fig. 4d), were observed, which were greatest in the first year following treatment; the differences tended to diminish over time, so that by year four the changes in the EBRT group had returned to baseline. The effectiveness of the $\mathrm{a}^{*}$ channel to capture skin colour changes due to radiotherapy in BCCT has already been reported [13]. Higher dissimilarity values in the $\mathrm{a}^{*}$ channel correspond to higher changes in the redness/greenness of the skin. This suggests that the main determinants of the difference in cosmetic outcome between the groups are asymmetry and colour (redness).

As there were only eight patients who received both IORT and EBRT in our study, their objective outcome assessment did not show any statistical significant result (see WEB Table 2).

\section{Discussion}

TARGIT is an international randomised clinical trial designed to test the hypothesis that the strategy of delivering a single dose of TARGIT to the tumour bed in patients eligible for breast conserving treatment is equivalent in terms of safety and effectiveness to a conventional course of whole breast external beam radiotherapy (EBRT) [4]. Cosmetic results have become an outcome of major importance following breast conserving surgery, since the 


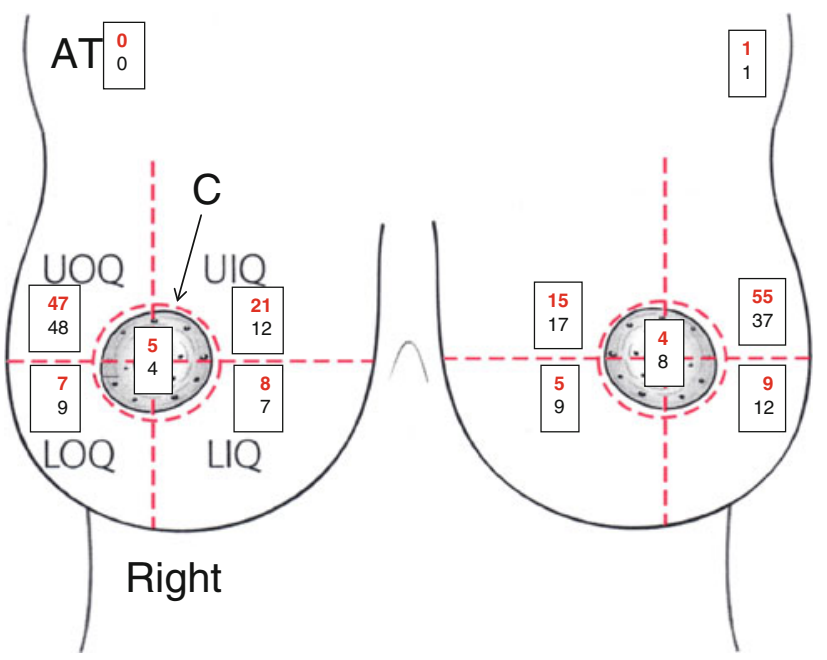

Key:

$\begin{array}{ll}\text { AT } & \text { Axillary tail } \\ \text { C } & \text { Central } \\ \text { UOQ } & \text { Upper Outer Quadrant } \\ \text { UIQ } & \text { Upper Inner Quadrant } \\ \text { LOQ } & \text { Lower Outer Quadrant } \\ \text { LIQ } & \text { Lower Inner Quadrant } \\ \text { Right } & \text { Right side }\end{array}$

TARGIT in red

EBRT in black

\begin{tabular}{|l|l|l|l|}
\hline Summary & Right & Left & Total \\
\hline TARGIT & $89 *$ & 89 & 178 \\
\hline EBRT & 80 & 84 & 164 \\
\hline & & & 342 \\
\hline
\end{tabular}

*includes one patient with missing quadrant data.

Fig. 3 Anatomical distribution of tumours

oncological outcome has proved to be similar to mastectomy in large randomised controlled trials [2, 3].

Conventional methods for aesthetic evaluation of breast conserving surgery include assessment of patient's appearance directly, or through photographs, by one or more observers. To date, there is no standardized objective tool to assess the cosmetic outcome of breast conserving treatment [14].

A couple of software tools have been recently applied in an effort towards objective evaluation of the surgical outcome; breast analyzing tool (BAT) and BCCT.core software. A relatively recent comparison of them on the same set of cases showed a similar performance on low-quality images and a superior performance of the BCCT.core software on higher quality images. This was attributed to the inclusion of colour and scar features [15].

Recent, as well as, ongoing trials evaluating the results of various radiotherapy protocols including intraoperative methods use subjective tools for assessing the aesthetic outcome [16-20]. Our objective evaluation indicates that cosmetic outcome of patients treated with IORT if given as a second procedure (post-pathology) in selected patients with low-risk breast cancer is significantly better than those treated with EBRT, a difference which can be seen by the first year (Fig. 4a). The difference continues into the 2nd year and from the 3rd year onwards the EBRT group catches up with the TARGIT group, which tends to remain at baseline levels. A possible explanation is that as intraoperative radiotherapy is delivered from within the breast the negative effect of radiotherapy to the skin and underlying breast tissue is therefore minimised. This result is in line with our previously reported findings of the TARGIT trial showing that radiation toxicity was more frequent in the EBRT group than in the TARGIT group [4].

Furthermore, it is known that radiation-induced changes in colour (e.g. dyspigmentation and telangiectasia) and shape (e.g. breast oedema and fibrosis) depend on the radiation dose as well as the irradiated volume [21]. The increased amount of breast tissue affected by radiotherapy in EBRT compared to the focal nature of IORT probably accounts for a higher degree of fibrosis which consequently leads to significant breast asymmetry (Fig. 4b).

Changes in the colour appear to be minimal with IORT, which can be explained by the minimal radiation dose reaching the skin [22]. Colour differences between the two protocols are more obvious one year following treatment and gradually decreases until year four when the difference disappears Fig. 4c. Interestingly, recently published results of a multicenter randomized trial comparing 3D conformal radiotherapy-accelerated partial breast irradiation (CRTAPBI) to standard whole breast irradiation show worse cosmetic outcome in patients treated with breast conserving surgery and APBI, attributed to the higher dose to skin with this external beam technique [23].

Scar visibility in BCCT.core is translated into local colour dissimilarity; the differences we have observed are similar to those of colour. EBRT reaches nonsignificant difference to IORT in scar appearance following year two (Fig. 4d). Considering that the specimen weight was not different between the two groups, the volume of tissue removed was not causing these differences in cosmetic outcome.

It is noteworthy that, surgeon's experience, tumour site or TARGIT IORT variables (e.g. applicator (obturator) size for delivery of the X-rays) do not vary between the two groups in the setting of this randomised controlled trial and they are not expected to affect the analysis.

One of the limitations of BCCT.core is that the assessment of texture is not possible which, in fact, is a problem for any tool based on photographs. Another possible drawback is the inability to evaluate in three dimensions. 
(a) Overall score

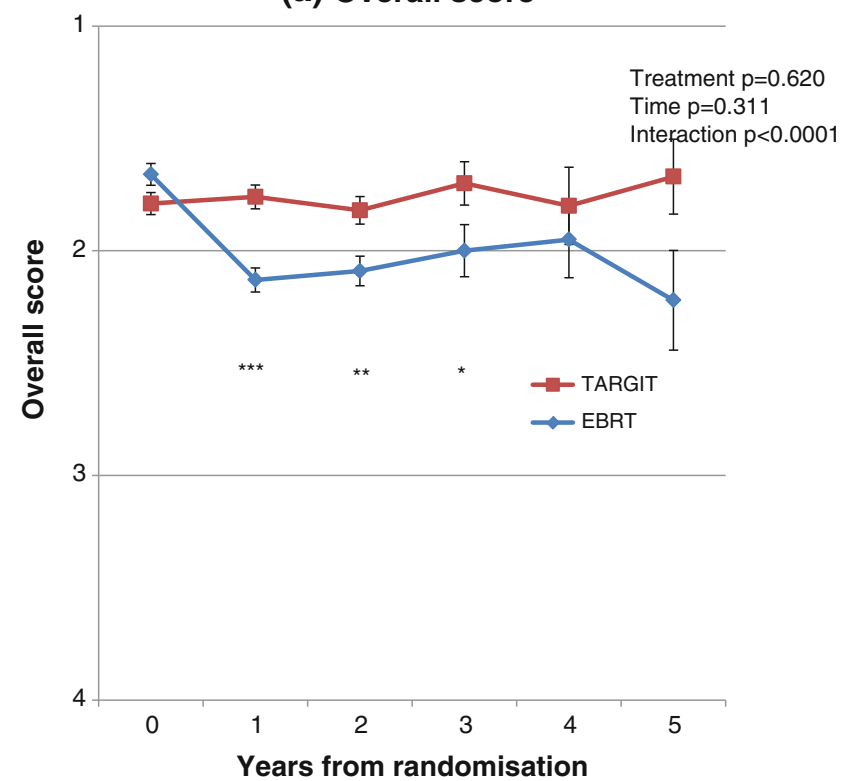

(c) Change in colour (cX2b)

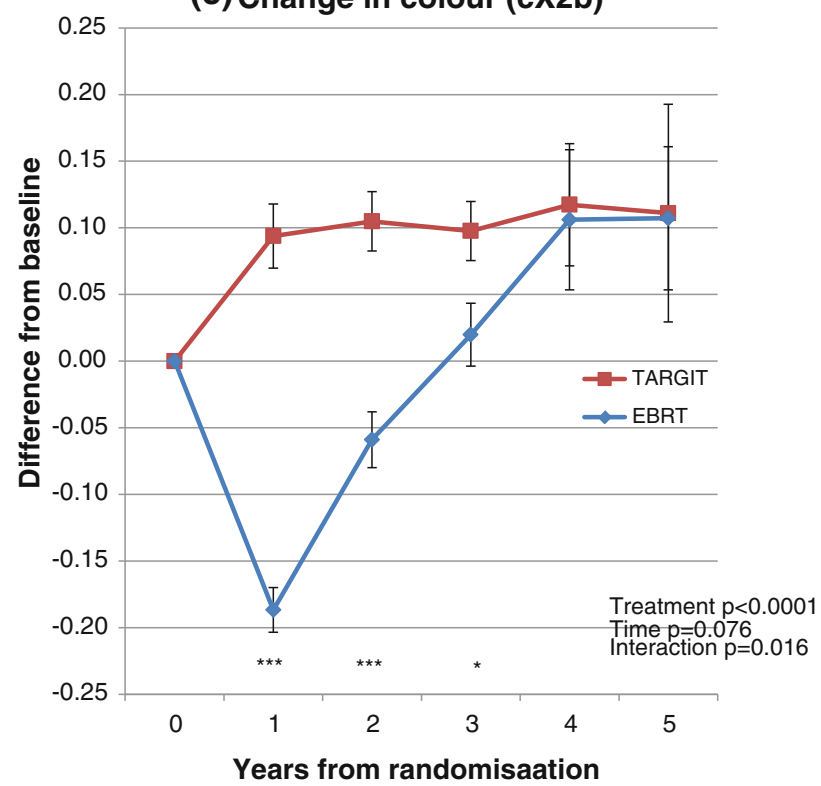

Fig. 4 Changes in objective cosmetic score from baseline. a Excellent 1, Good 2, Fair 3, Poor 4), b Changes lower breast contour (a measure of asymmetry) from baseline. A negative value indicates an increase in asymmetry, c Changes in colour (cX2b) from baseline. A negative value indicates more 'redness', d Changes in scar (sEMDb) from

3D evaluation of the aesthetic result has been recently suggested as a more precise method to quantify the effect of breast surgery [24-26]. Nevertheless, 3D software algorithms have not included features such as skin colour or scar appearance, which significantly influence the breast appearance overall [27]. We believe that inclusion of these parameters in software evaluating the aesthetic outcome of the breast following surgery and radiotherapy is a sine qua

\section{(b) Change in lower breast contour (pLBC)}

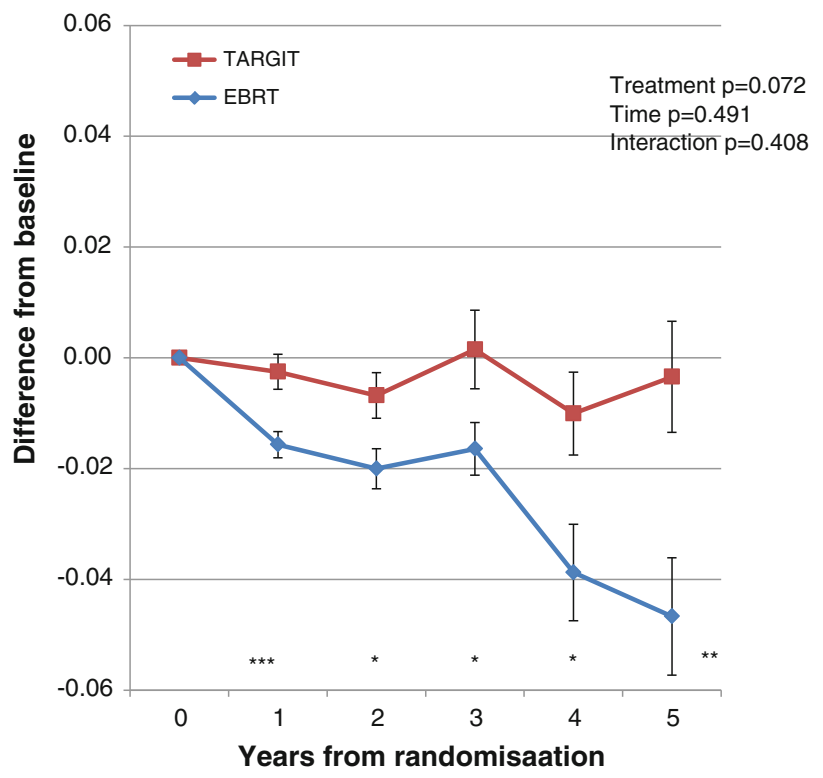

(d) Change in scar (sEMDb)

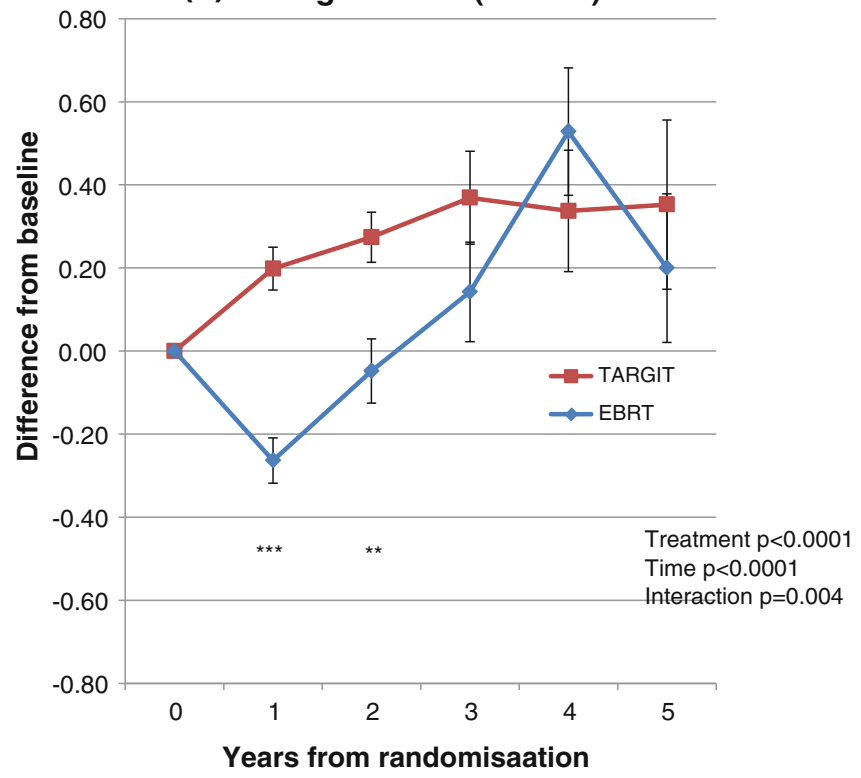

baseline. A negative value is associated with a more obvious scar. For each graph, the number of patients per treatment group at each year is as follows (Year, EBRT, IORT): 0,154,168; 1,146,154; 2,101,106; $3,35,40 ; 4,20,20 ; 5,9,9$

non condition. Our group is in the process of addressing these limitations.

A recent study evaluating late radiation toxicity after TARGIT has concluded that patients treated with IORT alone have about half the risk for developing higher grade toxicities as compared to standard whole breast radiotherapy [28]. This is the first study reporting an objective assessment of cosmesis in TARGIT with superior aesthetic outcome of 
TARGIT compared to EBRT, and further reinforces the advantages of using IORT in the treatment of selected women with early breast cancer.

In conclusion, this total objective assessment of the aesthetic outcome of patients in a randomised setting demonstrates that those treated with TARGIT have a superior cosmetic result to those patients who received conventional external beam radiotherapy.

Acknowledgments The main TARGIT A Trial is funded by the HTA (project ref 07/60/49) ISRCTN34086741 and sites in Australia were funded by a project grant awarded by the National Health and Medical Research Council. The authors thank the TARGIT Trialists' Group for the TARGIT A Trial.

Conflict of interest Carl Zeiss (the manufacturer of the Intrabeam system) covers travelling expenses for meetings of the TARGIT Trial International Steering Committee and conferences where a presentation about Targeted Intraoperative Radiotherapy is being made.

\section{References}

1. Forouzanfar MH, Foreman KJ, Delossantos AM et al (2011) Breast and cervical cancer in 187 countries between 1980 and 2010: a systematic analysis. Lancet 378:1461-1484

2. Veronesi U, Cascinelli N, Mariani L et al (2002) Twenty-year follow-up of a randomized study comparing breast-conserving surgery with radical mastectomy for early breast cancer. N Engl J Med 347:1227-1232

3. Fisher B, Anderson S, Bryant J et al (2002) Twenty-year followup of a randomized trial comparing total mastectomy, lumpectomy, and lumpectomy plus irradiation for the treatment of invasive breast cancer. N Engl J Med 347:1233-1241

4. Vaidya JS, Joseph DJ, Tobias JS et al (2010) Targeted intraoperative radiotherapy versus whole breast radiotherapy for breast cancer (TARGIT - a trial): an international, prospective, randomised, non-inferiority phase 3 trial. Lancet 376:91-102

5. Siegel R, Desantis C, Virgo K et al (2012) Cancer treatment and survivorship statistics, 2012. CA Cancer J Clin 62:220-241

6. Kurtz J (1995) Impact of radiotherapy on breast cosmesis. Breast 4:163-169

7. Pezner RD, Patterson MP, Hill LR et al (1985) Breast retraction assessment: an objective evaluation of cosmetic results of patients treated conservatively for breast cancer. Int $\mathrm{J}$ Radiat Oncol Biol Phys 11:575-578

8. Harris JR, Levene MB, Svensson G, Hellman S (1979) Analysis of cosmetic results following primary radiation therapy for stages I and II carcinoma of the breast. Int J Radiat Oncol Biol Phys 5:257-261

9. Fitzal F, Krois W, Trischler H et al (2007) The use of a breast symmetry index for objective evaluation of breast cosmesis. Breast 16:429-435

10. Cardoso MJ, Cardoso J, Amaral N et al (2007) Turning subjective into objective: the BCCT.core software for evaluation of cosmetic results in breast cancer conservative treatment. Breast 16:456-461

11. Cardoso JS, Cardoso MJ (2007) Towards an intelligent medical system for the aesthetic evaluation of breast cancer conservative treatment. Artif Intell Med 40:115-126
12. Taylor ME, Perez CA, Halverson KJ et al (1995) Factors influencing cosmetic results after conservation therapy for breast cancer. Int J Radiat Oncol Biol Phys 31:753-764

13. Oliveira HP, Magalhaes A, Cardoso MJ, Cardoso JS (2010) An accurate and interpretable model for BCCT.core. Conf Proc IEEE Eng Med Biol Soc 2010:6158-6161

14. Cardoso MJ, Magalhaes A, Almeida T et al (2008) Is face-only photographic view enough for the aesthetic evaluation of breast cancer conservative treatment? Breast Cancer Res Treat 112:565-568

15. Cardoso MJ, Cardoso JS, Wild T, Krois W, Fitzal F (2009) Comparing two objective methods for the aesthetic evaluation of breast cancer conservative treatment. Breast Cancer Res Treat 116:149-152

16. Barnett GC, Wilkinson JS, Moody AM et al (2011) Randomized controlled trial of forward-planned intensity-modulated radiotherapy for early breast cancer: interim results at 2 years. Int $\mathbf{J}$ Radiat Oncol Biol Phys 82:715-723

17. Ivanov O, Dickler A, Lum BY, Pellicane JV, Francescatti DS (2011) Twelve-month follow-up results of a trial utilizing Axxent electronic brachytherapy to deliver intraoperative radiation therapy for early-stage breast cancer. Ann Surg Oncol 18:453458

18. Lemanski C, Azria D, Gourgon-Bourgade S et al (2010) Intraoperative radiotherapy in early-stage breast cancer: results of the Montpellier phase II trial. Int J Radiat Oncol Biol Phys 76:698-703

19. Haley M, Beriwal S, Heron DE et al (2009) MammoSite accelerated partial breast irradiation: a single-institution outcomes analysis with 2 years of follow up. Brachytherapy 8:9-13

20. Sacchini V, Beal K, Goldberg J, Montgomery L, Port E, McCormick B (2008) Study of quadrant high-dose intraoperative radiation therapy for early-stage breast cancer. Br J Surg 95:1105-1110

21. Munshi A, Kakkar S, Bhutani R, Jalali R, Budrukkar A, Dinshaw KA (2009) Factors influencing cosmetic outcome in breast conservation. Clin Oncol (R Coll Radiol) 21:285-293

22. Eaton DJ, Best B, Brew-Graves C et al (2012) In vivo dosimetry for single-fractiontargeted intraoperative radiotherapy (TARGIT) for breast cancer. Int J Radiat Oncol Biol Phys 82:e819-e824

23. Olivotto IA, Whelan TJ, Parpia S, Kim DH, Berrang T, Truong PT, Kong I, Cochrane B, Nichol A, Roy I, Germain I, Akra M, Reed M, Fyles A, Trotter T, Perera F, Beckham W, Levine MN, Julian JA (2013) Interim cosmetic and toxicity results from RAPID: a randomized trial of accelerated partial breast irradiation using three-dimensional conformal external beam radiation therapy. J Clin Oncol. doi:10.1200/JCO.2013.50.5511

24. Losken A, Fishman I, Denson DD, Moyer HR, Carlson GW (2005) An objective evaluation of breast symmetry and shape differences using 3-dimensional images. Ann Plast Surg 55:571-575

25. Catanuto G, Spano A, Pennati A, Nava M (2009) Three-dimensional digital evaluation of breast symmetry after breast conservation therapy. J Am Coll Surg 208:166

26. Moyer HR, Carlson GW, Styblo TM, Losken A (2008) Three dimensional digital evaluation of breast symmetry after breast conservation therapy. J Am Coll Surg 207:227-232

27. Cardoso MJ, Cardoso JS, Vrieling C et al (2012) Recommendations for the aesthetic evaluation of breast cancer conservative treatment. Breast Cancer Res Treat 135:629-637

28. Sperk E, Welzel G, Keller A et al (2012) Late radiation toxicity after intraoperative radiotherapy (IORT) for breast cancer: results from the randomized phase III trial TARGIT A. Breast Cancer Res Treat 135:253-260 